\title{
Pisa syndrome in Parkinson's disease: diagnostic and management challenges
}

This article was published in the following Dove Press journal:

Journal of Parkinsonism and Restless Legs Syndrome

19 April 2016

Number of times this article has been viewed

\section{Vladimir Miletić}

Department of Neurology, Movement Disorders Centre, University Hospital Centre Zagreb, Zagreb, Croatia
Correspondence: Vladimir Miletić

Department of Neurology,

Movement Disorders Centre,

University Hospital Centre Zagreb,

Kišpatićeva 12, 10000 Zagreb, Croatia

Tel +385 I 2388345

Fax +385 I 2388377

Email vladimir.miletic@gmail.com
Abstract: Pisa syndrome is a rare clinical entity characterized by marked lateral flexion of the trunk, which is typically mobile and resolves at supine position. When observed in clinical practice, it denotes an incapacitating symptom of underlying neurodegenerative disease such as Parkinson's disease, multiple system atrophy, and Alzheimer's disease. The underlying pathophysiological mechanisms responsible for the development of Pisa syndrome are poorly understood, and its management remains a challenge. In this review, we will focus our attention on Pisa syndrome in patients with Parkinson's disease, and provide an update on prevalence, pathophysiology, clinical manifestation, and treatment options.

Keywords: Pisa syndrome, lateral trunk flexion, Parkinson's disease

\section{Introduction}

Pisa syndrome (PS) denotes marked lateral flexion of the trunk, which is typically mobile and resolves at supine position. It was originally described by Ekbom et $\mathrm{al}^{1}$ as acute truncal dystonia or pleurothonus related to butyrophenone administration. The term PS was subsequently applied to patients with dementia and other neurodegenerative diseases, such as Alzheimer's disease,${ }^{2}$ Lewy body disease, ${ }^{3}$ Parkinson's disease (PD),${ }^{4-7}$ multiple system atrophy, ${ }^{8}$ progressive supranuclear palsy, ${ }^{9}$ and Huntington's disease,${ }^{10}$ who developed lateral trunk deviation. In addition, PS has been described in patients with normotensive hydrocephalus, ${ }^{11}$ subdural hematoma, ${ }^{12}$ subacute sclerosing panencephalitis, ${ }^{13}$ or can present as an isolated and idiopathic condition. ${ }^{14}$ In PD patients, PS can occur either as a motor complication of the underlying neurodegenerative disorder or can be drug-induced. Practically, all dopaminergic drugs, including levodopa combinations (eg, levodopa/carbidopa, levodopa/benserazide, levodopa/carbidopa/entacapone), ${ }^{15,16}$ ergot derivative pergolide, ${ }^{4,15}$ nonergot derivatives pramipexole ${ }^{15}$ and ropinirole, ${ }^{17}$ and monoamine oxidase-B inhibitor rasagiline can cause PS. ${ }^{18}$ Other drugs associated with PS are typical and atypical antipsychotics, tricyclic antidepressants, selective serotonin reuptake inhibitors, antiemetic drugs, lithium, benzodiazepines, cholinesterase inhibitors, and valproic acid (Table 1). ${ }^{19-23}$ In this review, we will focus our attention on PS in patients with PD and provide an update on prevalence, pathophysiology, clinical manifestation, and treatment options.

$\mathrm{PD}$ is the second most common neurodegenerative disease and is characterized by bradykinesia, resting tremor, rigidity, and postural instability. ${ }^{24}$ It is a progressive, lifelong, and so far incurable disorder causing significant reduction of quality of life and increasing health care burden. Besides aforementioned classical motor symptoms, 
Table I Causes of Pisa syndrome

\begin{tabular}{l} 
Drugs \\
Antipsychotics (typical and atypical) \\
Tricyclic antidepressants \\
Selective serotonin reuptake inhibitors \\
Antiemetics \\
Lithium \\
Benzodiazepines \\
Cholinesterase inhibitors \\
Valproic acid \\
Levodopa combinations (eg, levodopa/carbidopa, levodopa/benserazide, \\
levodopa/carbidopa/entacapone) \\
Ergot derivative-pergolide \\
Nonergot derivatives-pramipexole and ropinirole \\
Monoamine oxidase-B inhibitor-rasagiline \\
Neurodegenerative diseases \\
Parkinson's disease \\
Multiple system atrophy \\
Progressive supranuclear palsy \\
Huntington's disease \\
Alzheimer's disease \\
Lewy body dementia \\
Other \\
Normotensive hydrocephalus \\
Subdural hematoma \\
Subacute sclerosing panencephalitis \\
Idiopathic form \\
\hline
\end{tabular}

additional aggravating conditions include postural abnormalities, which are quite common in this population. According to the results of a retrospective observational study, a third of patients with PD had a deformity of their limbs, neck, or trunk. ${ }^{25}$ Almost 2 centuries ago, in the fundamental paper An Essay on the Shaking Palsy, James Parkinson described distinctive stooped or bent posture of patients with PD. ${ }^{26}$ Nevertheless, significant proportion of patients exhibit more severe postural abnormalities or spinal alignment, leading to significant disability. These severe deformities include PS, camptocormia, scoliosis, and antecollis. ${ }^{27}$ It is noteworthy that most of the patients present with a combination of postural deformities. The underlying pathophysiology of these deformities is largely unknown, and their management remains a challenge.

\section{Epidemiology}

The lack of consistent diagnostic criteria could explain small number of epidemiological studies regarding prevalence of PS in PD patients. Most of the epidemiological data concerning frequency of PS arise from studies conducted in psychiatric population and are not going to be discussed here. Multicenter cross-sectional study, conducted in Italian movement disorders tertiary centers, systematically investigated the prevalence of PS and its relationship with clinical and demographic features in a large cohort of consecutive patients with PD. ${ }^{28}$ Among 1,631 enrolled patients with PD, PS was detected in 143 patients (prevalence 8.8\%). Patients with PS were older, had lower body mass index, longer disease duration, higher disease stages, poorer quality of life, more frequent falls as well as occurrence of "veering gait" (ie, the progressive deviation toward one side when patient walked forward and backward with eyes closed). Patients with PS received higher levodopa-equivalent daily dose and were more likely to be treated with a combination of levodopa and dopamine agonists. Osteoporosis and arthrosis were significantly the most frequently associated medical conditions in patients with PS. Another Italian single-center study detected 26 patients with lateral trunk deviation in the cohort of 1,400 patients with parkinsonism. ${ }^{29}$ Several studies investigated the prevalence of scoliosis in PD patients. Yet, the results are highly variable with prevalence of scoliosis ranging from $2 \%$ to $60 \%$ in PD patients. ${ }^{25,30,31}$ Still, it is important to emphasize that the terms "scoliosis" and "Pisa syndrome" are not synonyms. The term "scoliosis" should be reserved only for patients whose posture cannot be improved by passive movement or supine positioning, and who have radiological evidence of a structural curve with axial vertebral rotation that persists when the effect of gravity has been eliminated.

\section{Pathophysiology}

The pathophysiological mechanisms underlying PS in PD patients have not been fully explained. Two different hypotheses have been proposed: 1) central hypothesis consider PS as a consequence of basal ganglia dysfunction along with altered sensory-motor integration, and possibly exacerbation by dopaminergic treatment; 2) according to the peripheral hypothesis, PS occurs primarily due to an alteration of the musculoskeletal system, such as myopathy of the paraspinal muscles and soft tissue changes (Figure 1). ${ }^{27,32}$

Asymmetrical central process is likely to play a role in the tendency to lean to one particular side. Studies in a rat model showed that chemical degeneration of the nigrostriatal pathway produced substantial spontaneous trunk deviation toward the denervated side. ${ }^{33,34}$ Most of the clinical studies have also shown that patients lean away from the most affected side, which is consistent with animal studies. ${ }^{35-37}$ However, few investigators failed to replicate this finding. ${ }^{28,30,31}$ Another observation pointing to the involvement of central dopaminergic system is that PS can occur as a motor complication of PD. Kim et $\mathrm{al}^{7}$ reported two PD patients who developed PS during the "off" period with favorable response 


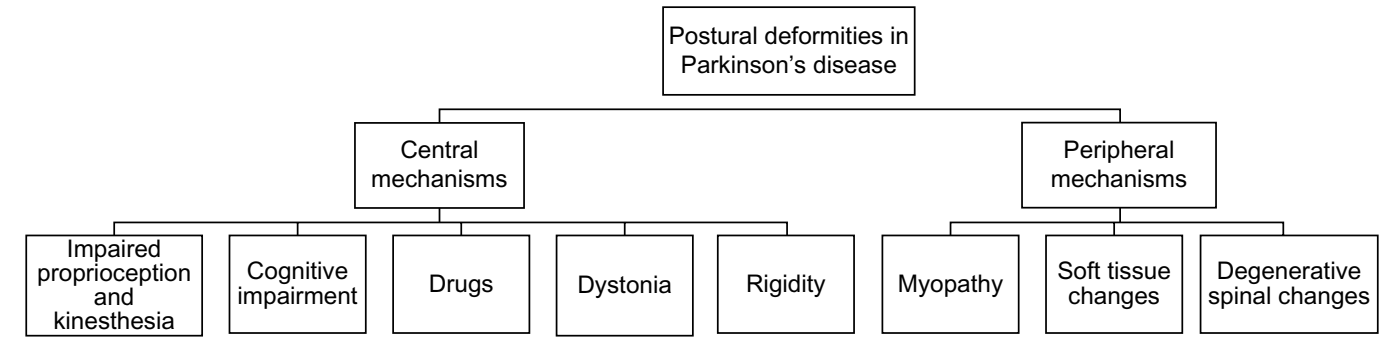

Figure I Pathophysiological mechanisms responsible for development of Pisa syndrome in Parkinson's disease.

to an increase of levodopa dosage. Nevertheless, most of the PD patients with PS will not benefit from increasing dopaminergic therapy. Moreover, PS is frequently reported as a side effect of almost all dopaminergic drugs. ${ }^{4,15-18}$ Together with other axial deformities, PS in PD patients usually represents therapy-resistant symptom, highlighting possible involvement of nondopaminergic pathways. ${ }^{38}$ Association of PS with cholinesterase inhibitors is well recognized. ${ }^{22}$ The improvement of PS with deep brain stimulation (DBS) of the pedunculopontine nucleus (PPN) suggests the contribution of the cholinergic system, and supports the hypothesis of the crucial role of dopaminergic-cholinergic imbalance in the pathophysiology of PS. ${ }^{21,39}$ Lateral trunk deviation can also occur following pallidotomy. There are two reports of PS developing in patients after pallidotomy, at a time interval of 4-9 years after surgery. ${ }^{40,41}$

Postural control depends on vestibular, visual, and somatosensory information. For proper postural control, all these components should act in highly harmonized, synchronous, and orchestral manner. Balance impairment in PD has recently been related to altered sensory-motor integration processing. ${ }^{42}$ Proprioception provides highly accurate information that helps to maintain body verticality. Several studies confirmed abnormality in proprioceptive function in PD patients. Vaugoyeau and Azulay ${ }^{43}$ showed that PD patients, unlike healthy subjects, were unable to maintain the vertical trunk orientation without visual compensation and were following the oscillations of the supporting platform, whereas the control subjects kept their body upright when deprived of visual cues and vestibular information. Perception of verticality is essential for postural control. Pereira et $\mathrm{a}^{44}$ showed that perception of verticality is affected in PD patients. This abnormal vertical perception together with disturbed processing of graviceptive pathways is associated with postural instability. Based on these results, Scocco et al ${ }^{45}$ investigated subjective visual verticality in PD patients with and without PS, and compared them with healthy controls. They found that the subjective visual verticality was altered in PD patients with and without PS when compared to healthy controls. The authors concluded that altered subjective visual verticality cannot be explained by intrinsic lateral deviation in PS patients, yet it must be secondary to either primary perceptual dysfunction or alterations of internal models of verticality. Until recently, it was thought that vestibular dysfunction is not involved in the pathogenesis of postural deformities affecting PD patients. ${ }^{46}$ However, recent investigation by Vitale et $\mathrm{a}^{17}{ }^{47}$ refuted this hypothesis. They evaluated vestibular function in eleven PD patients with lateral trunk flexion and in eleven age-, sex-, and disease duration-matched patients without lateral trunk flexion. A peripheral, unilateral vestibular hypofunction was identified in all patients with lateral trunk flexion. The vestibular hypofunction was ipsilateral to the leaning side and contralateral to the most affected parkinsonian side in all the patients. In the control group, seven subjects had no vestibular signs and four subjects had unilateral vestibular hypofunction without clinically evident lateral trunk flexion. Interestingly, two of the latter patients subsequently developed lateral trunk flexion ipsilateral to the vestibular deficit and contralateral to the side most affected by PD.

A complex relationship exists between postural control and cognition. Postural control requires a complex motorcognitive interaction where multiple sensorimotor processes occur in order to maintain balance and orientation in response to external stimuli. ${ }^{48} \mathrm{Up}$ to now, only one study investigated the possible contribution of cognitive dysfunction to occurrence of PS in PD patients. ${ }^{49}$ Among 40 PD patients enrolled, 20 had PS. All patients underwent comprehensive neuropsychological battery to assess behavioral disturbances, memory, attention, frontal/executive, and visuospatial functions. Results revealed a significant association of PS with altered attention and visuoperceptual functions, suggesting that the occurrence of PS may be associated with alteration of both frontal-striatal systems and posterior cortical areas.

Several electrophysiological studies using electromyography have been performed in PD patients with PS. The aim was to 1) investigate activation pattern of the axial paraspinal muscle and 2) find signs of possible denervation or myopathy. 
Tinazzi et $\mathrm{al}^{50}$ detected two different patterns of muscular activation: a hyperactivity of lumbar paraspinals ipsilateral to trunk bending side and hyperactivity of paraspinals contralateral to trunk bending side. These observations suggest that a dystonic activity could play a role in determining the bending ipsilaterally to PS and that the contralateral excessive muscle activation represents a compensatory mechanism. Tassorelli et $\mathrm{al}^{51}$ found an abnormal tonic hyperactivity on the side of the trunk's deviation in abdominal oblique muscles. The largest study conducted so far enrolled 60 PD patients with PS and showed asymmetric ability to generate maximal voluntary force of the external oblique muscles supporting a central desynchronization of axial muscles as a significant contributor for the bending of the spine in erect position. ${ }^{52}$ These results could have important implication to physiotherapy and the use of botulinum toxin (BTX) in the treatment of PS. So far conducted investigations failed to detect denervation and myopathy, underlining the importance of central dysfunction. However, the impact of degenerative spinal disease on soft tissue and muscle changes should not be disregarded. Some authors speculate that abnormal posture cannot be entirely explained by active dystonia and instead may reflect either intrinsic muscle and soft tissue changes resulting from a dystonia present earlier in the course of the disease or another more complex impairment of proprioceptive motor control in PD. ${ }^{53}$ Pathophysiology of PS in PD patients is very probably multifactorial, but the bulk of the data supports central, rather than peripheral hypotheses. ${ }^{32}$

\section{Diagnosis and clinical presentation}

There is no consensus on the diagnostic criteria for PS. Initially, Bonanni et $\mathrm{al}^{29}$ proposed the following definition for lateral axial dystonia: more than $15^{\circ}$ lateral flexion of the trunk, increasing during walking, not present when supine, and in the absence of any mechanical restriction to trunk movement (ie, degenerative spinal disease), with continuous electromyographic activity in the lumbar paraspinal muscles ipsilateral to the bending side. Doherty et $\mathrm{al}^{27}$ deviated from definition by Bonanni et $\mathrm{al}^{29}$ and proposed that a diagnosis of PS requires at least $10^{\circ}$ lateral flexion, which can be completely alleviated by passive mobilization or lying in a supine position. A reason for this deviation was that the pathophysiological mechanism of PS in PD patients may not be completely dystonic and should not require electrophysiological studies to define it.

PS can develop in acute (rapid deterioration within few days or weeks), subacute (followed by rapid deterioration over months), and chronic fashion (insidious at first with gradual worsening). ${ }^{15,54,55}$ Early recognition of PS represents mainstay of the treatment because chronic forms are often resistant to therapy. Most of the PD patients with PS are not aware of lateral trunk deviation in the early stage. ${ }^{35}$ At the beginning, only a slight tendency to lean on side can be observed while patient is sitting, with worsening during walking. A special phenomenon is "veering gait", which denotes progressive deviation toward one side when patient is walking forward and backward with eyes closed. In advance stage, patients often experience debilitating pain, dyspnea, or unsteadiness leading to falls.

Diagnosis of PS is based on clinical examination, but for the correct measurement of lateral deviation, use of goniometer is required. It is important to differentiate PS from scoliosis, which is defined as the lateral curvature of the spine with a Cobb angle of $10^{\circ}$ or more in the coronal plane. For this purpose, X-rays in standing and supine positions are necessary. Besides scoliosis, differential diagnosis of postural deformities in PD patients includes specific muscle disorders such as myasthenia gravis, focal myositis, and facioscapulohumeral dystrophy. ${ }^{56-58}$

\section{Treatment}

The lack of knowledge about principal pathophysiological mechanisms responsible for the development of postural deformities in patients with $\mathrm{PD}$ represents a major obstacle in therapeutic approach. Still, several useful tips could be of help in everyday clinical practice. In patients with acute or subacute development of lateral trunk deviation, it is mandatory to collect data about recent changes in medication. Early recognition and either withdrawal of offending agent or reintroduction of dopaminergic therapy can prevent veering toward the subchronic and chronic irreversible variant. ${ }^{15,54,59}$ In some PD patients, PS can appear as a motor complication during the "off" period. ${ }^{7}$ In such cases, increasing the dose of levodopa may provide amelioration of abnormal posture. Other oral medications used to treat PS include anticholinergics and novel antipsychotics without interference with dopaminergic receptors, such as clozapine and quetiapine. ${ }^{14,54,60}$ However, clinicians should be very precautious when prescribing atypical antipsychotics because they can induce and aggravate PS. ${ }^{61,62}$

Based on the assumption that PS is not a motor complication of $\mathrm{PD}$, rather due to dystonia of paraspinal muscles, application of BTX has been tried but with inconsistent results. In the blinded crossover study by Bonanni et al, ${ }^{29}$ nine PD patients with lateral axial dystonia who failed to improve from oral medications were randomly assigned into 
two groups; four patients received BTX and five placebo as a first treatment, and were switched to BTX or placebo in the following treatment session. Abobotulinum toxin A was injected under electromyographic control at four sites into the paraspinal muscles $2-2.5 \mathrm{~cm}$ lateral to spinous processes at level L2-L5 on the side of the trunk flexion, for a total dose of $500 \mathrm{U}$. None of the patients receiving the placebo experienced benefit. On the other hand, six patients treated with BTX showed an improvement between 50\% and $87.5 \%$. In one patient, only subjective benefit was reported, while two patients did not report any benefit. Up to now, there are no consensus guidelines on muscle selection for BTX application. Some authors suggest that injection in paraspinal muscles should be avoided, and priority should be given to infiltrating external oblique muscle. ${ }^{52}$ The most frequently injected muscles in the study conducted by Tassorelli et $\mathrm{al}^{63}$ were the iliopsoas and the rectus abdominis. Dupeyron et $\mathrm{l}^{64}$ reported new target muscle for BTX application. They described a complete and 1 year lasting resolution of PS after BTX injection in the quadratus lumborum muscle. Although no guidelines exist, all authors highlight the importance and necessity of electromyography in detecting hyperactive (rigid/dystonic) muscles before the administration of BTX. Tassorelli et $\mathrm{al}^{63}$ investigated whether the addition of BTX type A injection into hyperactive trunk muscles may improve the effectiveness of the rehabilitation program in PD patients with PS. Twenty-six PD patients were enrolled in the randomized, placebo-controlled trial. Group A was treated with BTX type A followed by a 4-week intensive program of conventional rehabilitation, while Group B received saline before 4-week rehabilitation. At the end of the rehabilitation period, both groups improved significantly in terms of static postural alignment and range of motion. Group A showed a significantly more marked reduction in pain score as compared with Group B, and a more prolonged efficacy on several clinical and kinematic variables. The authors suggested that BTX may be considered as an important addition to the rehabilitation program for PD subjects with PS for improving axial posture and trunk mobility, as well as for a better control of pain. Even without addition of BTX, sole 4-week trunk-specific rehabilitation treatment is shown to significantly improve lateral trunk flexion in PD. ${ }^{65}$ Furthermore, Frazzitta et $\mathrm{l}^{52}$ strongly support early rehabilitation before development of postural deformities, with special emphasis on stretching exercises for the external oblique and paraspinal muscles.

Besides oral medications, BTX application, and conventional rehabilitation, treatment with DBS of PPN has also been tried. Shih et $\mathrm{al}^{39}$ described the beneficial effect of DBS of the PPN contralateral to the most affected side and contralateral to the trunk leaning in a PD patient with PS. In another case, stimulators were implanted in the PPN ipsilateral to the side of the trunk leaning. ${ }^{66}$ Unfortunately, the initial improvement was not retained over time, probably reflecting the complex pathophysiology of PS. The reason for PS improvement with either ipsilateral or contralateral DBS is probably related to the diffuse bilateral projections of PPN. Further studies investigating the impact of PPN DBS



Figure 2 Treatment algorithm for Pisa syndrome in Parkinson's disease. Abbreviations: PS, Pisa syndrome; PD, Parkinson's disease; DBS, deep brain stimulation. 
on PS are needed for obtaining final conclusion. Treatment options for PS in PD are summarized in Figure 2.

\section{Conclusion}

PS is common, but not inevitable in PD patients. Having significant impact on daily functioning and quality of life, PS deserves special attention and should not be overlooked. Exact pathophysiological mechanism is still poorly understood, but it seems unlikely that a single pathological process is responsible for the occurrence of PS. More complex interplay between central basal ganglia dysfunction, together with proprioceptive disintegration and altered cognitive processing, is usually required for the development of PS, thus highlighting central rather than peripheral hypotheses of PS origin. Still, peripheral mechanisms should not be ignored and future investigations should determine their role in the pathophysiology of PS. For further studies with intention to gather epidemiological data and to delineate pathophysiological mechanisms, consensus on diagnostic criteria is mandatory. Treatment options for PS include oral medication such as anticholinergics, or dopaminergic drugs if PS denotes motor complication of PD. Rehabilitation with emphasis on stretching exercises is recommended from the moment of the diagnosis of PD. Association of PS with specific cognitive dysfunctions supports the idea of cognitive rehabilitation programs aiming to improve cognitive capacity and, in turn, ameliorate postural abnormality. Development of standardized protocols for cognitive and physical rehabilitation is necessary for adequate treatment, and future studies should address this issue. Application of BTX provided promising results, especially when combined with conventional rehabilitation procedures. However, further studies are required to clarify which muscles are the best targets. Although favorable effects of DBS on PS have been reported in previous case reports, the surgical outcomes of DBS are not predictable and require further investigations.

Probably, the most important thing when dealing with PS in PD patients is early recognition of this rare and incapacitating symptom, because appropriate management in acute or subacute phase can prevent chronic irreversible state.

\section{Disclosure}

The author reports no conflicts of interest in this work.

\section{References}

1. Ekbom K, Lindholm H, Ljungberg L. New dystonic syndrome associated with butyrophenone therapy. Z Neurol. 1972;202(2):94-103.

2. Vanacore N, Suzzareddu G, Maggini M, Casula A, Capelli P, Raschetti R. Pisa syndrome in a cohort of Alzheimer's disease patients. Acta Neurol Scand. 2005;111(3):199-201.
3. Shinfuku M, Nakajima S, Uchida H, Watanabe K, Kocha H, Kashima H. Pisa syndrome caused by an acetylcholinesterase inhibitor in a patient with dementia with Lewy bodies. Psychiatry Clin Neurosci. 2011;65(3):299.

4. Cannas A, Solla P, Floris G, Borghero G, Tacconi P, Spissu A. Reversible Pisa syndrome in Parkinson's disease during treatment with Pergolide: a case report. Clin Neuropharm. 2005;28(5):252-253.

5. Gambarin M, Antonini A, Moretto G, et al. Pisa syndrome without neuroleptic exposure in a patient with Parkinson's disease: case report. Mov Disord. 2006;21(2):270-273.

6. Harada K. Pisa syndrome without neuroleptic exposure in a patient with Parkinson's disease: a case report. Mov Disord. 2006;21(12):2264-2265.

7. Kim JS, Park JW, Chung SW, Kim YI, Kim HT, Lee KS. Pisa syndrome as a motor complication of Parkinson's disease. Parkinsonism Relat Disord. 2007;13(2):126-128.

8. Colosimo C. Pisa syndrome in a patient with multiple system atrophy. Mov Disord. 1998;13(3):607-609.

9. Solla P, Cannas A, Costantino E, Orofino G, Lavra L, Marrosu F. Pisa syndrome in a patient with progressive supranuclear palsy. J Clin Neurosci. 2012;19(6):922-923.

10. Salazar Z, Tschopp L, Calandra C, Micheli F. Pisa syndrome and parkinsonism secondary to valproic acid in Huntington's disease. Mov Disord. 2008;23(16):2430-2431.

11. Leon-Sarmiento FE, Pradilla G, Del Rosario Zambrano M. Primary and reversible Pisa syndrome in juvenile normal pressure hydrocephalus. Acta Neuropsychiatr. 2013;25(1):57-60.

12. Marchione P, Spallone A, Valente M, Giannone C, De Angelis F, Meco G. Reversible Pisa syndrome associated to subdural haematoma: case report. BMC Neurol. 2014;14:149.

13. Malhotra HS, Garg RK. Pearls and Oy-sters: Pisa syndrome: an unusual feature of adult-onset fulminant SSPE. Neurology. 2015;84(3):e12-e14.

14. Bhattacharya KF, Giannakikou I, Munroe N, et al. Primary anticholinergic-responsive Pisa syndrome. Mov Disord. 2000;15(6):1285-1287.

15. Cannas A, Solla P, Floris G, et al. Reversible Pisa syndrome in patients with Parkinson's disease on dopaminergic therapy. $J$ Neurol. 2009;256(3):390-395.

16. Solla P, Cannas A, Congia S, et al. Levodopa/carbidopa/entacaponeinduced acute Pisa syndrome in a Parkinson's disease patient. J Neurol Sci. 2008;275(1-2):154-156.

17. Galati S, Möller JC, Städler C. Ropinirole-induced Pisa syndrome in Parkinson disease. Clin Neuropharmacol. 2014;37(2):58-59.

18. Fasano A, Di Matteo A, Vitale C, et al. Reversible Pisa syndrome in patients with Parkinson's disease on rasagiline therapy. Mov Disord. 2011;26(14):2578-2580.

19. Suzuki T, Matsuzaka H. Drug-induced Pisa syndrome (pleurothotonus): epidemiology and management. CNS Drugs. 2002;16(3):165-174.

20. Stübner S, Padberg F, Grohmann R, et al. Pisa syndrome (pleurothotonus): report of a multicenter drug safety surveillance project. $J$ Clin Psychiatry. 2000;61(8):569-574

21. Villarejo A, Camacho A, García-Ramos R, et al. Cholinergicdopaminergic imbalance in Pisa syndrome. Clin Neuropharmacol. 2003;26(3):119-121.

22. Zannas AS, Okuno Y, Doraiswamy PM. Cholinesterase inhibitors and Pisa syndrome: a pharmacovigilance study. Pharmacotherapy. 2014;34(3):272-278.

23. Yohanan M,Aulakh JS, Weith J, et al. Pisa syndrome in a patient in a wheelchair taking valproic acid. Am J Psychiatry. 2006;163(2):325-326.

24. Litvan I, Bhatia KP, Burn DJ, et al. Movement Disorders Society Scientific Issues Committee report: SIC Task Force appraisal of clinical diagnostic criteria for Parkinsonian disorders. Mov Disord. 2003;18(5):467-486.

25. Ashour R, Jankovic J. Joint and skeletal deformities in Parkinson's disease, multiple system atrophy, and progressive supranuclear palsy. Mov Disord. 2006;21(11):1856-1863.

26. Parkinson J. An Essay on the Shaking Palsy. London: Whittingham and Rowland; 1817. 
27. Doherty KM, van de Warrenburg BP, Peralta MC, et al. Postural deformities in Parkinson's disease. Lancet Neurol. 2011;10(6):538-549.

28. Tinazzi M, Fasano A, Geroin C, et al. Pisa syndrome in Parkinson disease: an observational multicenter Italian study. Neurology. 2015;85(20):1769-1779.

29. Bonanni L, Thomas A, Varanese S, Scorrano V, Onofrj M. Botulinum toxin treatment of lateral axial dystonia in Parkinsonism. Mov Disord. 2007;22(14):2097-2103

30. Baik JS, Kim JY, Park JH, Han SW, Park JH, Lee MS. Scoliosis in patients with Parkinson's disease. J Clin Neurol. 2009;5(2):91-94.

31. Grimes JD, Hassan MN, Trent G, Halle D, Armstrong GW. Clinical and radiographic features of scoliosis in Parkinson's disease. Adv Neurol. 1987;45:353-355.

32. Castrioto A, Piscicelli C, Pérennou D, Krack P, Debû B. The pathogenesis of Pisa syndrome in Parkinson's disease. Mov Disord. 2014;29(9):1100-1107.

33. Ungerstedt U, Butcher LL, Butcher SG, Anden NE, Fuxe K. Direct chemical stimulation of dopaminergic mechanisms in the neostriatum of the rat. Brain Res. 1969;14(2):461-471.

34. Herrera-Marschitz M, Utsumi H, Ungerstedt U. Scoliosis in rats with experimentally-induced hemiparkinsonism: dependence upon striatal dopamine denervation. J Neurol Neurosurg Psychiatry. 1990;53(1):39-43.

35. Duvoisin RC, Marsden CD. Note on the scoliosis of Parkinsonism. J Neurol Neurosurg Psychiatry. 1975;38(8):787-793.

36. Indo T, Ando K. Studies on the scoliosis of Parkinsonism. Rinsho Shinkeigaku. 1980;20(1):40-46.

37. Di Matteo A, Fasano A, Squintani G, et al. Lateral trunk flexion in Parkinson's disease: EMG features disclose two different underlying pathophysiological mechanisms. J Neurol. 2011;258(5):740-745.

38. Vorovenci RJ, Biundo R, Antonini A. Therapy-resistant symptoms in Parkinson's disease. J Neural Transm. 2016;123(1): $19-30$.

39. Shih LC, Vanderhorst VG, Lozano AM, Hamani C, Moro E. Improvement of pisa syndrome with contralateral pedunculopontine stimulation. Mov Disord. 2013;28(4):555-556.

40. van de Warrenburg BP, Bhatia KP, Quinn NP. Pisa syndrome after unilateral pallidotomy in Parkinson's disease: an unrecognised, delayed adverse event? J Neurol Neurosurg Psychiatry. 2007;78(3): 329-330.

41. Spanaki C, Zafeiris S, Plaitakis A. Levodopa-aggravated lateral flexion of the neck and trunk as a delayed phenomenon of unilateral pallidotomy. Mov Disord. 2010;25(5):655-656.

42. Benatru I, Vaugoyeau M, Azulay JP. Postural disorders in Parkinson's disease. Neurophysiol Clin. 2008;38(6):459-465.

43. Vaugoyeau M, Azulay JP. Role of sensory information in the control of postural orientation in Parkinson's disease. J Neurol Sci. 2010;289(1-2):66-68

44. Pereira CB, Kanashiro AK, Maia FM, Barbosa ER. Correlation of impaired subjective visual vertical and postural instability in Parkinson's disease. J Neurol Sci. 2014;346(1-2):60-65.

45. Scocco DH, Wagner JN, Racosta J, Chade A, Gershanik OS. Subjective visual vertical in Pisa syndrome. Parkinsonism Relat Disord. 2014;20(8):878-883.

46. Pastor MA, Day BL, Marsden CD. Vestibular induced postural responses in Parkinson's disease. Brain. 1993;116(Pt 5):1177-1190.

Journal of Parkinsonism \& Restless Legs Syndrome

\section{Publish your work in this journal}

Journal of Parkinsonism and Restless Legs Syndrome is an online, open access, peer-reviewed journal. The journal publishes review articles, historical reviews, original research articles, case reports, letters to the editor, clinical teaching cases, neuroradiology highlights, neuropathology highlights, neuropsychiatry highlights, autobiographies, conference
47. Vitale C, Marcelli V, Furia T, et al. Vestibular impairment and adaptive postural imbalance in parkinsonian patients with lateral trunk flexion. Mov Disord. 2011;26(8):1458-1463.

48. Horak FB. Postural orientation and equilibrium: what do we need to know about neural control of balance to prevent falls? Age Ageing. 2006;35(Suppl 2):ii7-ii11.

49. Vitale C, Falco F, Trojano L, et al. Neuropsychological correlates of Pisa syndrome in patients with Parkinson's disease. Acta Neurol Scand. Epub 2015 Oct 2. doi: 10.1111/ane.12514.

50. Tinazzi M, Juergenson I, Squintani G, et al. Pisa syndrome in Parkinson's disease: an electrophysiological and imaging study. $J$ Neurol. 2013;260(8):2138-2148.

51. Tassorelli C, Furnari A, Buscone S, et al. Pisa syndrome in Parkinson's disease: clinical, electromyographic, and radiological characterization. Mov Disord. 2012;27(2):227-235.

52. Frazzitta G, Balbi P, Gotti F, et al. Pisa syndrome in Parkinson's disease: electromyographic aspects and implication for rehabilitation. Parkinsons Dis. 2015;2015:437190. doi: 10.1155/2015/437190.

53. Doherty KM, Davagnanam I, Molloy S, Silveira-Moriyama L, Lees AJ. Pisa syndrome in Parkinson's disease: a mobile or fixed deformity? J Neurol Neurosurg Psychiatry. 2013;84(12):1400-1403.

54. Miletić V, Radić B, Relja M. Acute pisa syndrome as a neurological emergency. J Neuropsychiatry Clin Neurosci. 2015;27(2):e159-e160.

55. Yokochi F. Lateral flexion in Parkinson's disease and Pisa syndrome J Neurol 2006;253(Suppl 7):VII17-VII20.

56. Abboud H, Sivaraman I, Ontaneda D, Tavee J. Camptocormia and Pisa syndrome as manifestations of acute myasthenia gravis exacerbation. J Neurol Sci. 2015;359(1-2):8-10.

57. Wunderlich S, Csoti I, Reiners K, et al. Camptocormia in Parkinson's disease mimicked by focal myositis of the paraspinal muscles. Mov Disord. 2002;17(3):598-600.

58. Doherty KM, Silveira-Moriyama L, Giladi N, Bhatia KP, Parton M, Lees AJ. Camptocormia: don't forget muscle disease in the movement disorder clinic. J Neurol. 2012;259(8):1752-1754.

59. Michel SF, Oscar AC, Correa TE, Alejandro PL, Micheli F. Pisa syndrome. Clin Neuropharmacol. 2015;38(4):135-140.

60. Arora M, Praharaj SK, Sarkar S. Clozapine effective in olanzapineinduced Pisa syndrome. Ann Pharmacother. 2006;40(12):2273-2275.

61. Kurtz G, Kapfhammer HP, Peuker B. Pisa syndrome in clozapine therapy. Nervenarzt. 1993;64(11):742-746.

62. Walder A, Greil W, Baumann P. Drug-induced Pisa syndromeunder quetiapine. Prog Neuropsychopharmacol Biol Psychiatry. 2009;33(7):1286-1287.

63. Tassorelli C, De Icco R, Alfonsi E, et al. Botulinum toxin type A potentiates the effect of neuromotor rehabilitation of Pisa syndrome in Parkinson disease: a placebo controlled study. Parkinsonism Relat Disord. 2014;20(11):1140-1144.

64. Dupeyron A, Viollet E, Coroian F, Gagnard C, Renard D, Castelnovo G Botulinum Toxin-A for treatment of Pisa syndrome: a new target muscle. Parkinsonism Relat Disord. 2015;21(6):669-670.

65. Bartolo M, Serrao M, Tassorelli C, et al. Four-week trunk-specific rehabilitation treatment improves lateral trunk flexion in Parkinson's disease. Mov Disord. 2010;25(3):325-331.

66. Ricciardi L, Piano C, Bentivoglio AR, Fasano A. Long-term effects of pedunculopontine nucleus stimulation for Pisa syndrome. Parkinsonism Relat Disord. 2014;20(12):1445-1446.

\section{Dovepress}

proceedings, abstracts and book reviews. The manuscript management system is completely online and includes a very quick and fair peerreview system, which is all easy to use. Visit http://www.dovepress.com/ testimonials.php to read real quotes from published authors. 